\title{
Descentralização da gestão: caminho percorrido, nós críticos e perspectivas
}

\author{
Decentralization of health management: \\ the path traveled to date, problematic points and prospects
}

Elisangela Pinafo ${ }^{1}$

Brígida Gimenez Carvalho ${ }^{2}$

Elisabete de Fátima Polo de Almeida Nunes ${ }^{2}$

${ }^{1}$ Centro de Ciências Biológicas - Campus Luiz Meneghel, Universidade Estadual do Norte do Paraná. Rodovia BR 369Km/54, Vila Maria. 86360-000 Bandeirantes PR Brasil. elisangelapinafo@ yahoo.com.br

${ }^{2}$ Programa de PósGraduação em Saúde Coletiva, Universidade Estadual de Londrina. Londrina PR Brasil.

\begin{abstract}
The scope of this study was to analyze the decentralization of health management at city level and its implications for comprehensive access and care for users. A "scoping review" located 1,902 references. After being subjected to the criteria of inclusion and exclusion by judges, 27 articles were selected and charted. The research revealed the following issues: what it was possible to establish by the process; problematic points in charting the path and prospects of decentralization. The following aspects were identified: increased access to primary care through increasing the number of professionals and family health teams; planning, evaluation and inter-sector aspects were seen as problematic; planning and management instruments are limited, and cities face difficulties in relation to medium and high complexity care. Funding is still a barrier to the advancement of decentralization, few studies have analyzed the guarantee of access to medium and high complexity care and studies that define the reality of small cities are needed. Several challenges were identified, such as the need for implementation of the regionalization process, shared management and an inter-federative network that ensures comprehensive access to health care.

Key words Decentralization, Health management, Unified Health System, Universal access to health care services, Comprehensive health care
\end{abstract}

Resumo Objetivou-se analisar a descentralização da gestão em saúde para o ente municipal e suas implicações para o acesso e a atenção integral ao usuário. Por meio de "scoping review" encontrou-se 1.902 referências. Submetidas aos critérios de inclusão e exclusão, por juízes, selecionou-se e mapeou-se 27 artigos. Do mapeamento resultaram os temas: o que foi possivel consolidar com o processo; nós críticos para trilhar o caminho e perspectivas para a descentralização. Verificou-se: ampliação do acesso à atenção básica com aumento do número de profissionais e de equipes de Saúde da Família; planejamento, avaliação e intersetorialidade foram tidos como problemáticos; instrumentos de planejamento e gestão são limitados, e os municípios encontram dificuldades na referência para a atenção de média e alta complexidade. O financiamento ainda é uma barreira para o avanço da descentralização, poucos estudos analisaram a garantia de acesso para média e alta complexidade e estudos que retratem a realidade dos municípios de pequeno porte são necessários. Vários desafios foram apontados como a necessidade de efetivação do processo de regionalização, gestão compartilhada e de rede interfederativa que garanta acesso e atenção integral à saúde.

Palavras-chave Descentralização, Gestão em saúde, Sistema Único de Saúde, Acesso universal aos serviços de saúde, Atenção integral à saúde 


\section{Introdução}

A descentralização da gestão do Sistema Único de Saúde (SUS) merece ser discutida na perspectiva do que ocorre em âmbito local para sua efetivação, e desta forma refletir sobre a realidade enfrentada pelos municípios brasileiros.

As normatizações federais para o processo de descentralização foram editadas e substituídas ao longo dos anos. De acordo com o Ministério da Saúde, nos anos de 1990 foram publicadas quatro Normas Operacionais Básicas (NOB), em 2001 a Norma Operacional da Assistência à Saúde (NOAS, reformulada em 2002), em 2006 o Pacto pela Saúde e, em 2011 o Decreto 7.508¹. Com todo este aparato legal, nota-se a passagem de um sistema centralizado para um modelo de governos municipais, com importante ganho de autonomia no campo da saúde 2 .

No entanto, as condições para que a descentralização atenda a garantia do acesso universal às ações e serviços de saúde e da atenção integral de acordo com as necessidades e demandas da população não estão asseguradas, apresentando resultados contraditórios no território nacional ${ }^{3}$.

Observam-se características heterogêneas dos sistemas descentralizados de saúde, que refletem as diferentes capacidades financeiras, administrativas e operacionais para a prestação da atenção à saúde e as distintas disposições políticas de governadores e prefeitos ${ }^{4}$.

A dificuldade em apresentar um planejamento regional das estratégias de descentralização compromete sua adequação às múltiplas realidades brasileiras. Sem considerar o papel das esferas estaduais a descentralização acentua as atribuições dos municípios na provisão de serviços ${ }^{2}$.

Com todas as transformações, desafios e autonomia política demandadas para os municípios brasileiros, o que se questiona hoje é a capacidade da esfera municipal em gerir as políticas públicas locais de forma eficiente ${ }^{5}$, diante de um contexto de subfinanciamento do SUS.

Como decorrência da heterogeneidade do Brasil, a mudança na alocação de recursos advinda da implementação da descentralização não tem sido suficiente para modificar o padrão de desigualdade no acesso aos bens e serviços de saúde ${ }^{6}$. Nesse processo, os municípios se vêem diante da tarefa de gerir uma rede de serviços heterogênea e não integrada institucionalmente ${ }^{7}$ e com oferta insuficiente de serviços na média complexidade ${ }^{8}$.
O presente estudo trata-se de uma scoping review que objetivou analisar o processo de descentralização da gestão em saúde ocorrida para o ente municipal e suas implicações para a garantia do acesso e da atenção integral ao usuário.

\section{Procedimento Metodológico}

Para alcançar os objetivos propostos, realizou-se um "scoping review" ou "scoping study". Trata-se de um tipo de revisão muito utilizada a partir de 2005, apresentada pelas professoras Hilary Arksey e Lisa O'Malley e compreende um conjunto de técnicas com finalidade de sumarizar o conhecimento sobre um dado tema de investigação. Este estudo seguiu as etapas previstas em um scoping review: (1) identificação da questão da pesquisa; (2) identificação dos estudos relevantes; (3) seleção dos estudos; (4) mapeamento dos dados; (5) confrontação, resumo e relato dos resultados.

O problema que disparou esta revisão foi a necessidade de sistematizar o conhecimento produzido sobre a descentralização do SUS, suas implicações para os municípios na atualidade e desafios que estes enfrentam para a garantia do acesso e da atenção integral aos usuários. Desta indagação, identificou-se a seguinte questão de pesquisa: Como se deu o processo de descentralização da gestão do SUS para os municípios e quais as implicações deste processo para a garantia do acesso e da atenção integral em saúde para os usuários?

Os critérios de inclusão foram: idioma: português, inglês e espanhol; período de publicação: a partir de 1993, tendo em vista que o processo de descentralização da gestão inicia-se formalmente com a NOB 93 até o período de Agosto de 2014 (período que ocorreu o término da busca das referências); objeto de estudo: implicações que a descentralização da gestão em saúde trouxe para o ente municipal; disponibilidade: resumo online com acesso gratuito. Como critérios de exclusão: livro ou capítulo de livro; dissertações, monografias e teses; documentos ministeriais e estudos tipo revisão.

A busca bibliográfica foi realizada nas seguintes bases de dados: Literatura Latino-Americana e do Caribe em Ciências da Saúde - Lilacs, United States National Library of Medicine at the National Institutes of Health - PubMed, Scientific Eletronic Library Online - SciELO, Publius Ovidius Naso - OVID, e WEB SAÚDE. 
Para a busca dos artigos nas bases de dados selecionadas, utilizou-se os Descritores em Ciências da Saúde-DeCS: regionalização, regional health planning, descentralização, decentralization, gestão em saúde, health management, acesso universal aos serviços de saúde, universal access to health care services; acessibilidade aos serviços de saúde, health services accessibility, sistema único de saúde, unified health system, políticas públicas de saúde, health public policies, atenção integral à saúde, comprehensive health care.

Os unitermos foram disponibilizados da seguinte forma para sua busca: descentralização and gestão em saúde; descentralização and sistema único de saúde; descentralização and regionalização; descentralização and acesso universal aos serviços de saúde; descentralização and acessibilidade aos serviços de saúde; descentralização and políticas públicas de saúde; descentralização and atenção integral à saúde. A busca ocorreu no período de 15 de julho a 14 de Agosto de 2014.

A seleção dos estudos relevantes foi realizada por meio de uma análise aplicada por dois juízes (o examinador padrão que é o autor principal deste estudo e o examinador I que é o segundo autor), de acordo com a questão norteadora e os critérios de inclusão e exclusão previamente definidos. Todos os estudos identificados pela estratégia de busca foram inicialmente avaliados pelo examinador padrão, por meio da análise dos títulos e resumos.

Houve seleção inicial de 1902 referências nas seguintes bases de dados: PubMed: 1440, Lilacs: 289, SciELO: 26; OVID: 47, WEB SAÚDE: 100. Após lidos os títulos e os resumos pelo primeiro juiz, este apontou 1832 referências classificadas como "não relacionadas ao tema" e 70 como "relacionadas ao tema”. Estes 70 artigos foram lidos na íntegra pelos dois juízes, sendo que o primeiro elegeu 17 e o segundo 20. Após comparação das duas análises verificou-se que os 17 artigos eleitos pelo primeiro juiz foram também eleitos pelo segundo. Houve discordâncias para os três artigos restantes, e para estes casos foi aplicada a técnica de consenso, na qual os dois juízes verificaram conjuntamente se estes respondiam à pergunta de pesquisa, sendo discutido e chegado à conclusão que mais dois seriam selecionados para a pesquisa, totalizando 19 estudos. Como forma de ampliar o levantamento bibliográfico, foi realizada busca manual nas referências dos estudos primários, pela qual foram identificados mais 15 , lidos na íntegra pelos dois juízes. Destes, oito artigos foram selecionados por ambos os juízes porque respondiam à pergunta de pesquisa. Dessa forma, o presente scoping review foi composto por 27 artigos.

O mapeamento de dados dos estudos foi realizado e as informações extraídas abrangeram: as referências do artigo, com local e ano de publicação, título do artigo, autores, formação dos autores, local de publicação, palavras chave, objetivos, metodologia, principais resultados, conclusões dos estudos. Em seguida, foram confrontados e discutidos os resultados para uma análise mais aprofundada dos estudos selecionados.

\section{Resultados}

\section{Caracterização dos estudos incluídos no scoping review}

Conhecer o contexto em que as pesquisas foram produzidas auxilia na compreensão da temática estudada. Nesta seção serão apresentadas algumas características dos estudos analisados.

Os artigos foram publicados a partir do ano de 2002, sendo 29,7\% (9) em 2010, e aumento da publicação a partir de 2007.

Quanto aos periódicos que publicaram sobre a temática foram: 9 (33,3\%) nas Revistas Ciência \& Saúde Coletiva e Cadernos de Saúde Pública; $2(7,4 \%)$ nas Revistas Saúde Soc e Revista Baiana de Saúde Pública; 1 (3,7\%) em cada um dos seguintes periódicos: Revista de Saúde Pública, Revista Latino-Americana de Enfermagem, Revista Enfermagem UERJ, Revista de Administração Pública, Revista Panamericana de Salud Pública. Os descritores mais encontrados foram: descentralização (17), Gestão em saúde (9), SUS (7) e Política de saúde (6).

O Quadro 1 traz a descrição dos trabalhos que fizeram parte do estudo.

Os estudos foram feitos por pesquisadores de todas as regiões do país. A região Sudeste teve predomínio com $13(48,1 \%)$ estudos (7 SP, 4 RJ e $2 \mathrm{MG})$, a região Nordeste com $11(40,8 \%)$ (8 $\mathrm{BA}, 1 \mathrm{PE}$ e $2 \mathrm{RN})$ e a região Sul (SC), Centro-Oeste (MT) e Norte (AM) apresentaram 1, (3,7\%) respectivamente.

O tamanho dos municípios estudados variou, $9(33,3 \%)$ artigos analisaram todos os portes de município, $9(33,3 \%)$ os de grande porte, 7 $(25,9 \%)$ não relataram o porte do município estudado e 2 (7,5\%) de médio porte. Apesar dos municípios de pequeno porte representarem aproximadamente $74 \%$ dos municípios brasileiros, não foram identificados estudos específicos para estes. 
Quadro 1. Descrição dos estudos utilizados pela scoping review.

\begin{tabular}{|c|c|c|c|c|}
\hline $\begin{array}{c}\text { Ano de } \\
\text { publicação }\end{array}$ & Revista & Local do estudo & Autores & Título do Artigo \\
\hline 2002 & RAP & $\begin{array}{l}\text { Município de Natal, } \\
\text { RN. }\end{array}$ & $\begin{array}{l}\text { Borges DF; } \\
\text { Fernandes KC }\end{array}$ & $\begin{array}{l}\text { A descentralização das ações e } \\
\text { serviços de saúde do SUS na cidade } \\
\text { de Natal: democratização ou } \\
\text { privatização? }\end{array}$ \\
\hline 2002 & $\begin{array}{l}\text { Ciência \& } \\
\text { Saúde Coletiva }\end{array}$ & $\begin{array}{l}1.643 \text { munic. de } \\
\text { diferentes regiões } \\
\text { brasileiras }\end{array}$ & $\begin{array}{l}\text { Arretche M; } \\
\text { Marques E }\end{array}$ & $\begin{array}{l}\text { Municipalização da saúde no Brasil: } \\
\text { diferenças regionais, poder do voto } \\
\text { e estratégias de governo. }\end{array}$ \\
\hline 2002 & $\begin{array}{l}\text { Ciência \& } \\
\text { Saúde Coletiva }\end{array}$ & $\begin{array}{l}\text { Agregados de } \\
\text { municípios Brasileiros } \\
\text { por porte populacional } \\
\text { ou inserção regional }\end{array}$ & $\begin{array}{l}\text { Costa NR; Pinto } \\
\text { LF }\end{array}$ & $\begin{array}{l}\text { Avaliação de programa de atenção à } \\
\text { saúde: incentivo à oferta de atenção } \\
\text { ambulatorial e a experiência da } \\
\text { descentralização no Brasil }\end{array}$ \\
\hline 2002 & $\begin{array}{l}\text { Ciência \& } \\
\text { Saúde Coletiva }\end{array}$ & $\begin{array}{l}\text { Município de São } \\
\text { Gonçalo, RJ. }\end{array}$ & $\begin{array}{l}\text { Monnerat GL; } \\
\text { Senna MCM; } \\
\text { Souza RG }\end{array}$ & $\begin{array}{l}\text { A reorganização dos serviços de } \\
\text { saúde no cenário local }\end{array}$ \\
\hline 2004 & $\begin{array}{l}\text { Ciência \& } \\
\text { Saúde Coletiva }\end{array}$ & $\begin{array}{l}\text { Município de Feira de } \\
\text { Santana, BA. }\end{array}$ & $\begin{array}{l}\text { Juliano IA; Assis, } \\
\text { MMA }\end{array}$ & $\begin{array}{l}\text { A vigilância sanitária em Feira } \\
\text { de Santana no processo de } \\
\text { descentralização da saúde (1998- } \\
\text { 2000) }\end{array}$ \\
\hline 2007 & $\begin{array}{l}\text { Caderno Saúde } \\
\text { Pública }\end{array}$ & Municípios da Bahia. & $\begin{array}{l}\text { Vieira-da-Silva } \\
\text { LM; Hartz ZMA; } \\
\text { Chaves SCL; Silva } \\
\text { GAP; Paim JS }\end{array}$ & $\begin{array}{l}\text { Análise da implantação da gestão } \\
\text { descentralizada em saúde: estudo } \\
\text { comparado de cinco casos na Bahia, } \\
\text { Brasil }\end{array}$ \\
\hline 2007 & $\begin{array}{l}\text { Caderno Saúde } \\
\text { Pública }\end{array}$ & $\begin{array}{l}\text { Dois municípios da } \\
\text { Bahia. }\end{array}$ & $\begin{array}{l}\text { Chaves SCL; } \\
\text { Vieira-da-Silva } \\
\text { LM }\end{array}$ & $\begin{array}{l}\text { Atenção à saúde bucal e a } \\
\text { descentralização da saúde no Brasil: } \\
\text { estudo de dois casos exemplares no } \\
\text { Estado da Bahia }\end{array}$ \\
\hline 2008 & $\begin{array}{l}\text { Rev. Enferm. } \\
\text { UERJ }\end{array}$ & $\begin{array}{l}\text { Um município da } \\
\text { Bahia. }\end{array}$ & $\begin{array}{l}\text { Souza MKB; } \\
\text { Melo CMM }\end{array}$ & $\begin{array}{l}\text { Perspectiva de enfermeiras gestoras } \\
\text { acerca da gestão municipal da saúde }\end{array}$ \\
\hline 2009 & $\begin{array}{l}\text { Rev. Baiana de } \\
\text { Saúde Pública }\end{array}$ & $\begin{array}{l}11 \text { municípios da } \\
\text { Bahia. }\end{array}$ & $\begin{array}{l}\text { Barros SG; } \\
\text { Vianna MIP; } \\
\text { Chaves SCL }\end{array}$ & $\begin{array}{l}\text { Descentralização da saúde e } \\
\text { utilização de serviços odontológicos } \\
\text { em } 11 \text { municípios da Bahia }\end{array}$ \\
\hline 2009 & $\begin{array}{l}\text { Caderno Saúde } \\
\text { Pública }\end{array}$ & $\begin{array}{l}\text { Município de São } \\
\text { Paulo, SP. }\end{array}$ & $\begin{array}{l}\text { Spedo SM; } \\
\text { Tanaka OY; Pinto } \\
\text { NRS }\end{array}$ & $\begin{array}{l}\text { O desafio da descentralização } \\
\text { do Sistema Único de Saúde em } \\
\text { município de grande porte: o caso } \\
\text { de São Paulo, Brasil }\end{array}$ \\
\hline 2009 & $\begin{array}{l}\text { Caderno Saúde } \\
\text { Pública }\end{array}$ & $\begin{array}{l}\text { Município de São } \\
\text { Paulo, SP. }\end{array}$ & $\begin{array}{l}\text { Pinto NRS; } \\
\text { Tanaka OY; } \\
\text { Spedo SM }\end{array}$ & $\begin{array}{l}\text { Política de saúde e gestão no } \\
\text { processo de (re)construção do SUS } \\
\text { em município de grande porte: um } \\
\text { estudo de caso de São Paulo, Brasil }\end{array}$ \\
\hline
\end{tabular}




\begin{tabular}{|c|c|c|c|c|}
\hline \multicolumn{5}{|c|}{ Quadro 1. continuação } \\
\hline $\begin{array}{c}\text { Ano de } \\
\text { publicação }\end{array}$ & Revista & Local do estudo & Autores & Título do Artigo \\
\hline 2009 & $\begin{array}{l}\text { Rev. Saúde } \\
\text { Pública }\end{array}$ & $\begin{array}{l}\text { Dois municípios de } \\
\text { SP, um do RJ, PB, BA, } \\
\text { respectivamente }\end{array}$ & $\begin{array}{l}\text { Scatena LM; Vila } \\
\text { TCSS; Ruffino } \\
\text { Netto A. et al. }\end{array}$ & $\begin{array}{l}\text { Dificuldades de acesso a serviços } \\
\text { de saúde para diagnóstico de } \\
\text { tuberculose em municípios do } \\
\text { Brasil }\end{array}$ \\
\hline 2010 & $\begin{array}{l}\text { Ciência \& } \\
\text { Saúde Coletiva }\end{array}$ & $\begin{array}{l}\text { Município de Juazeiro } \\
\text { do Norte, CE. }\end{array}$ & $\begin{array}{l}\text { Coriolano MWL; } \\
\text { Albuquerque } \\
\text { GA; Araújo NS; } \\
\text { Oliveira MA; } \\
\text { Lima MM. }\end{array}$ & $\begin{array}{l}\text { Vivenciando o processo de } \\
\text { municipalização do SUS no } \\
\text { município de Juazeiro do Norte } \\
\text { (CE) }\end{array}$ \\
\hline 2010 & $\begin{array}{l}\text { Saúde } \\
\text { Sociedade }\end{array}$ & $\begin{array}{l}\text { Município de São } \\
\text { Paulo, SP. }\end{array}$ & $\begin{array}{l}\text { Spedo SM; Pinto } \\
\text { NRS; Tanaka OY }\end{array}$ & $\begin{array}{l}\text { A regionalização intermunicipal do } \\
\text { Sistema Único de Saúde (SUS): um } \\
\text { estudo de caso do município de São } \\
\text { Paulo-SP, Brasil. }\end{array}$ \\
\hline 2010 & $\begin{array}{l}\text { Ciência \& } \\
\text { Saúde Coletiva }\end{array}$ & $\begin{array}{l}\text { Municípios do Mato } \\
\text { Grosso. }\end{array}$ & $\begin{array}{l}\text { Marangon MS; } \\
\text { Scatena JHG; } \\
\text { Costa EA }\end{array}$ & $\begin{array}{l}\text { Vigilância sanitária: estratégias } \\
\text { para sua descentralização em Mato } \\
\text { Grosso, 1996-2005 }\end{array}$ \\
\hline 2010 & $\begin{array}{l}\text { Rev. } \\
\text { Panamericana } \\
\text { de Salud } \\
\text { Publica }\end{array}$ & $\begin{array}{l}\text { Amostra de } \\
\text { municípios do Brasil. }\end{array}$ & $\begin{array}{l}\text { Fleury S; } \\
\text { Uverney ALM; } \\
\text { Kronemberger } \\
\text { TSK; Zani FB }\end{array}$ & $\begin{array}{l}\text { Governança local no sistema } \\
\text { descentralizado de saúde no Brasil }\end{array}$ \\
\hline 2010 & $\begin{array}{l}\text { Saúde e } \\
\text { Sociedade }\end{array}$ & $\begin{array}{l}\text { Cinco municípios da } \\
\text { Amazônia. }\end{array}$ & $\begin{array}{l}\text { Vieira JMR; } \\
\text { Garnelo L; } \\
\text { Hortale V A }\end{array}$ & $\begin{array}{l}\text { Análise da Atenção Básica em Cinco } \\
\text { Municípios da Amazônia Ocidental, } \\
\text { com Ênfase no Programa Saúde da } \\
\text { Família }\end{array}$ \\
\hline 2010 & $\begin{array}{l}\text { Caderno Saúde } \\
\text { Púbica }\end{array}$ & $\begin{array}{l}\text { Um município da } \\
\text { Bahia }\end{array}$ & $\begin{array}{l}\text { Cunha } \mathrm{ABO} \\
\text { Vieira-da-Silva } \\
\text { LM }\end{array}$ & $\begin{array}{l}\text { Acessibilidade aos serviços de saúde } \\
\text { em um município do Estado da } \\
\text { Bahia, Brasil, em gestão plena do } \\
\text { sistema }\end{array}$ \\
\hline 2010 & $\begin{array}{l}\text { Caderno Saúde } \\
\text { Pública }\end{array}$ & $\begin{array}{l}\text { Municípios de Aracajú } \\
\text { (SE), Belo Horizonte } \\
\text { (MG), Florianópolis } \\
\text { (SC) e Vitória (ES). }\end{array}$ & $\begin{array}{l}\text { Almeida PF; } \\
\text { Giovanella } \\
\text { L; Mendonça } \\
\text { MHM; Escorel S }\end{array}$ & $\begin{array}{l}\text { Desafios à coordenação dos } \\
\text { cuidados em saúde: estratégias de } \\
\text { integração entre níveis assistenciais } \\
\text { em grandes centros urbanos }\end{array}$ \\
\hline 2010 & $\begin{array}{l}\text { Caderno Saúde } \\
\text { Pública }\end{array}$ & $\begin{array}{l}\text { Dois municípios da } \\
\text { Bahia. }\end{array}$ & $\begin{array}{l}\text { Barreto JL; } \\
\text { Guimarães MCL }\end{array}$ & $\begin{array}{l}\text { Avaliação da gestão descentralizada } \\
\text { da assistência farmacêutica básica } \\
\text { em municípios baianos, Brasil }\end{array}$ \\
\hline 2010 & $\begin{array}{l}\text { Rev. Baiana de } \\
\text { Saúde Pública }\end{array}$ & $\begin{array}{l}\text { Dois municípios da } \\
\text { Bahia. }\end{array}$ & $\begin{array}{l}\text { Molesini JA; } \\
\text { Formigli VLA; } \\
\text { Guimarães MCL; } \\
\text { Melo CMM }\end{array}$ & $\begin{array}{l}\text { Programação pactuada integrada e } \\
\text { gestão compartilhada do SUS }\end{array}$ \\
\hline 2011 & $\begin{array}{l}\text { Caderno Saúde } \\
\text { Pública }\end{array}$ & $\begin{array}{l}\text { Município de } \\
\text { Campinas, SP. }\end{array}$ & $\begin{array}{l}\text { Balista SRR; } \\
\text { Santiago SM; } \\
\text { Corrêa Filho HR }\end{array}$ & $\begin{array}{l}\text { A descentralização da vigilância da } \\
\text { saúde do trabalhador no Município } \\
\text { de Campinas, São Paulo, Brasil: uma } \\
\text { avaliação do processo }\end{array}$ \\
\hline
\end{tabular}




\begin{tabular}{|c|c|c|c|c|}
\hline \multicolumn{5}{|c|}{ Quadro 1. continuação } \\
\hline $\begin{array}{c}\text { Ano de } \\
\text { publicação }\end{array}$ & Revista & Local do estudo & Autores & Título do Artigo \\
\hline 2011 & $\begin{array}{l}\text { Rev. } \\
\text { Latino-Am. } \\
\text { Enfermagem }\end{array}$ & $\begin{array}{l}\text { Nove municípios de } \\
\text { MG }\end{array}$ & $\begin{array}{l}\text { Lanza FM; Lana } \\
\text { FCF }\end{array}$ & $\begin{array}{l}\text { Descentralização das ações } \\
\text { de controle da hanseníase na } \\
\text { microrregião de Almenara, Minas } \\
\text { Gerais }\end{array}$ \\
\hline 2012 & $\begin{array}{l}\text { Ciência \& } \\
\text { Saúde Coletiva, }\end{array}$ & $\begin{array}{l}14 \text { municípios do Rio } \\
\text { Grande do Norte. }\end{array}$ & $\begin{array}{l}\text { Leite VR; Lima } \\
\text { KC; Vasconcelos } \\
\text { CM }\end{array}$ & $\begin{array}{l}\text { Financiamento, gasto público e } \\
\text { gestão dos recursos em saúde: o } \\
\text { cenário de um estado brasileiro }\end{array}$ \\
\hline 2013 & $\begin{array}{l}\text { Saúde e } \\
\text { Sociedade }\end{array}$ & $\begin{array}{l}\text { Município de Itapira, } \\
\text { SP. }\end{array}$ & $\begin{array}{l}\text { Vieira V; } \\
\text { Andrade FR; } \\
\text { Castro CGJ; } \\
\text { Bighetti TI; } \\
\text { Narvai PC }\end{array}$ & $\begin{array}{l}\text { Municipalização de serviços de } \\
\text { saúde segundo profissionais de } \\
\text { saúde bucal em um município do } \\
\text { interior do estado de São Paulo, } \\
\text { Brasil }\end{array}$ \\
\hline 2013 & $\begin{array}{l}\text { Ciência \& } \\
\text { Saúde Coletiva }\end{array}$ & $\begin{array}{l}\text { Municípios de } \\
\text { Diamantina, Gouveia e } \\
\text { Datas, MG. }\end{array}$ & $\begin{array}{l}\text { Viegas SMF; } \\
\text { Penna CMM }\end{array}$ & $\begin{array}{l}\text { O SUS é universal, mas vivemos de } \\
\text { cotas }\end{array}$ \\
\hline 2014 & $\begin{array}{l}\text { Ciência \& } \\
\text { Saúde Coletiva }\end{array}$ & $\begin{array}{l}\text { Município de } \\
\text { Florianópolis, SC. }\end{array}$ & $\begin{array}{l}\text { Mello ALSF; } \\
\text { Andrade SR; } \\
\text { Moysés SJ; } \\
\text { Erdmann AL }\end{array}$ & $\begin{array}{l}\text { Saúde bucal na rede de atenção e } \\
\text { processo de regionalização }\end{array}$ \\
\hline
\end{tabular}

Em sua maioria, 17 (63,0\%) pesquisas, abordaram o impacto da descentralização sobre a gestão da saúde no município de forma geral analisando vantagens, dificuldades e avanços, entre outros; $4(14,8 \%)$ analisaram a área da Saúde Bucal, 2 (7,4\%) a Vigilância Sanitária, e 1 (3,7\%) estudo analisou cada uma das seguintes áreas: Vigilância do Trabalhador, Tuberculose, Hanseníase e Assistência Farmacêutica.

$\mathrm{Na}$ análise dos estudos, os temas gerais que melhor caracterizaram o que havia emergido foram: O que foi possível consolidar com o processo da descentralização; Nós críticos para trilhar o caminho da descentralização e ao final foram tecidas algumas considerações sobre perspectivas da descentralização.

\section{O que foi possível consolidar com o processo da descentralização}

A gestão do SUS é vista como um processo complexo, permeada por um contexto cujas influências políticas tanto locais, estaduais, quanto federais determinam as práticas de saúde, e interferem nas relações de poder e nas tomadas de decisões ${ }^{10}$. Nesta seção estão apresentados os resultados referentes às vantagens e aos avanços do processo de descentralização da gestão em saúde, analisando o percurso construído pelos municípios para a concretização da municipalização do SUS no cotidiano.

Constatou-se o fortalecimento da municipalização, da capacidade de governo do nível local, reconhecendo-se muitas vantagens trazidas pelo SUS, no sentido de que os municípios ficaram mais preparados para assistir a população ${ }^{11-15}$.

A ampliação do acesso em nível local e da cobertura assistencial em relação às ações básicas de saúde foram vistas como pontos positivos, bem como, o aumento do número de municípios com estabelecimentos básicos ou ambulatoriais de saúde em todas as regiões brasileiras ${ }^{10,12,13,16,17}$.

Estudo realizado em agregados de municípios brasileiros identificou que a existência de uma Unidade Básica de Saúde (UBS) é um resultado importante da descentralização, especialmente 
para aqueles com população inferior a 5.000 habitantes, pois foram os que mais se beneficiaram com a ampliação da oferta de atenção básica ${ }^{16}$.

Investimentos foram feitos para a expansão da rede básica, visando ampliar a oferta da atenção primária. Essa iniciativa partiu da preocupação com a disponibilização de serviços básicos, objetivando facilitar seu acesso, ampliar sua cobertura e impactar na situação de saúde da população $^{7,12,16}$.

No município de Juazeiro do Norte (CE), em municípios da Bahia e em cinco municípios da Amazônia, após a introdução dos trabalhos da Estratégia Saúde da Família (ESF) houve ampliação da cobertura populacional e observaram-se melhorias nos indicadores de saúde da atenção básica $^{12-14}$.

Em um município da Bahia os resultados foram positivos quanto à ESF, pois com as Unidades de Saúde da Família (USF) verificou-se melhor desempenho em relação às UBS tradicionais, dada a existência de estratégias organizacionais da assistência como a presença de lista de espera, de estratégias para o acolhimento e da melhor organização do sistema de referência e contrarreferência. O desempenho da acessibilidade geográfica obteve classificação satisfatória para as USF, fruto da estratégia de descentralização que, além da ampliação no número de unidades, obedece a critérios de territorialização para sua implantação ${ }^{18}$.

Em cinco municípios da Amazônia Ocidental a ESF foi identificada como uma política preferencial de continuidade das gestões municipais, por ser uma prioridade do Ministério da Saúde, com estímulo financeiro vinculado ${ }^{14}$. No Município de São Paulo, ampliou-se a cobertura populacional e o número de equipes de Saúde da Família (SF), no entanto, ainda necessita-se de esforços para atingir a cobertura prevista por esta política de saúde ${ }^{19}$.

Outros avanços se relacionaram aos secretários e conselhos municipais de saúde que tiveram maior influência na elaboração do orçamento e na definição de prioridades ${ }^{12,13}$. Também a prática de prestação de contas foi institucionalizada ${ }^{20}$. Assim, cada vez mais a sociedade passa a receber informações incluindo a prestação de contas da gestão, a partir de diferentes mecanismos e meios.

A capacidade de governo no âmbito local, vinculada ao perfil do gestor e dos quadros técnicos, pode ter contribuído para um melhor desempenho nas práticas assistenciais, como também a capacitação e a adesão dos profissionais de saúde para as políticas públicas ${ }^{12,20}$.
Quanto à força de trabalho no SUS, identificou-se crescimento do número de profissionais de nível superior atuando na atenção básica e ambulatorial em todo o país, com ampliação na composição multifuncional dos empregos (não médico). Outro dado importante é que quanto menor o município, maior foi o número de empregos criados. Com o aumento da rede de atenção, do número de profissionais e do incentivo financeiro por meio do $\mathrm{PAB}$, houve ampliação do acesso aos serviços de saúde de forma geral no âmbito local ${ }^{16}$.

Os municípios brasileiros de até $80 \mathrm{mil} \mathrm{ha-}$ bitantes responderam de modo responsável aos incentivos da descentralização. No Brasil, os que mais se beneficiaram com a redistribuição de recursos promovida pelo Ministério da Saúde através do $\mathrm{PAB}$ foram os que possuíam população inferior a 50.000 habitantes. Em contrapartida, cerca de $30 \%$ das cidades com mais de 50.000 habitantes tiveram o montante de recursos reduzidos $^{16}$.

Outro ponto positivo observado no desenvolvimento da atenção básica foram iniciativas de interação com a média e a alta complexida$\mathrm{de}^{6,12,21}$. Estudo realizado em Aracaju (SE), Belo Horizonte (MG), Florianópolis (SC) e Vitória (ES) identificou iniciativas de articulação entre a Atenção Primária à Saúde (APS) e os demais níveis assistenciais. $\mathrm{O}$ desenvolvimento de instrumentos de coordenação clínica como protocolos de acesso da APS à atenção especializada, representam estratégias que visam à garantia de atenção integral por parte da rede básica ${ }^{21}$.

Outro estudo verificou que a ampliação do acesso da atenção básica aos serviços de alta e média complexidade foi facilitada para um município que, à época de realização do estudo ${ }^{12}$, estava habilitado na gestão plena do sistema pela NOB 96. A NOB/96 alterou as modalidades de gestão local para duas formas: a plena da atenção básica e a plena do sistema de saúde. Na primeira modalidade, os municípios passam a responder totalmente pela assistência ambulatorial, pelas ações básicas de vigilância sanitária e epidemiológica e pela gerência de todas as unidades básicas de saúde existentes na região. $\mathrm{Na}$ segunda, os municípios, além da atenção básica, passam a responder integralmente por todas as ações referentes aos serviços do SUS em sua área de abrangência, inclusive pela oferta de procedimentos de média e alta complexidade tecnológica ${ }^{7}$. Entre os pontos positivos para a ampliação do acesso aos serviços de outros níveis de atenção foram citados nesse estudo ${ }^{12}$ : a governabilidade e o apoio 
político formal que o gestor municipal possuía e a autonomia da Secretaria Municipal de Saúde em relação à utilização dos recursos financeiros do SUS. Esse contexto possivelmente propiciou a reorganização da rede assistencial com maior controle sobre o setor privado, com redução das internações desnecessárias e ênfase na atenção básica com ampliação da cobertura a partir de ações programáticas e melhoria da acessibilidade com ampliação de central de marcação de consultas.

A interação entre os níveis assistenciais e os municípios de diversos portes foi feita por meio da organização de Consórcios Intermunicipais de Saúde de forma regionalizada, com a participação e a interação entre os municípios de menor porte com os de médio ou grande porte. Esta proposta do Consórcio Intermunicipal foi vista como um importante passo para a garantia do acesso a outros serviços e de interação entre os municípios e os serviços que são prestados por eles, pois pode permitir a articulação entre os diferentes níveis de atenção, possibilitando a formulação de ações integrais em saúde por meio da regionalização $0^{6}$.

Quanto aos estudos de áreas específicas como saúde bucal, do trabalhador e vigilância sanitária, verificou-se que a municipalização impulsionou a organização dos serviços e o modo de produção do cuidado.

Em relação aos serviços odontológicos, houve qualificação dos cuidados básicos e ampliação da efetividade e eficiência do sistema público de saúde, com expansão da utilização dos serviços ambulatoriais ${ }^{15,22,23}$. A APS foi vista como porta de entrada para o acesso à atenção odontológica, cuja ampliação foi proporcionada, na maioria das vezes, pela responsabilização dos profissionais e pelo vínculo com a comunidade. A garantia de acesso aos Centros de Especialidades Odontológicas (CEO) e/ou Unidades de Pronto Atendimento (UPA/24h), que prestam serviços de urgência odontológica, proporcionaram continuidade da assistência, organização da atenção à saúde, responsabilização, sem romper com a linha de cuidados da saúde bucal ${ }^{23}$.

Quanto à área de saúde do trabalhador, foi identificado o fortalecimento do sistema municipal descentralizado, que valoriza a rede básica e coloca a descentralização da vigilância em saúde nesta área como perspectiva para todas as unidades. Como facilidades pontuaram o aumento da capacidade técnica e a integração entre as equipes. Este processo apontou que aproximando trabalhadores e usuários, a descentralização iria ao encontro do princípio da integralidade da atenção, respondendo às necessidades de saúde das pessoas ${ }^{24}$.

Nos artigos analisados referente às áreas de Hanseníase, Tuberculose, Vigilância Sanitária e Assistência Farmacêutica não foram apontados aspectos positivos sobre o processo de descentralização.

\section{Nós críticos para trilhar o caminho da descentralização}

Nesta seção estão apontadas as desvantagens e as dificuldades encontradas na trajetória do processo de descentralização do SUS em nível municipal.

Estudos realizados em municípios da Bahia referiram que a descentralização sozinha não explica o estágio de organização do sistema municipal de saúde, e que as práticas de gestão e as assistenciais são áreas críticas que necessitam de intervenções referentes ao planejamento, avaliação e na intersetorialidade ${ }^{10,12}$.

Em 14 municípios do Rio Grande do Norte, observou-se que o financiamento do SUS é garantido por transferência federal e receitas municipais, com pouca participação de recursos estaduais. Apesar da relevância do recurso federal, a implementação das políticas de saúde é viabilizada pela maior participação dos municípios no financiamento. No entanto, ainda que tenha diminuído a participação do governo federal no financiamento da saúde pública, sua presença não foi reduzida na determinação de políticas em nível de atenção básicaa ${ }^{25}$.

A implementação de políticas definidas pelas esferas federal e estadual foi vista como ponto negativo, pois o município se sente compelido a ampliar seus serviços para obter maior repasse, pois estas políticas estão vinculadas a incentivos financeiros. Isso aumenta a responsabilidade do município, principalmente em relação ao comprometimento do orçamento municipal para além do previsto na legislação ${ }^{10}$. Em contrapartida, outro estudo discute que a ampliação dos gastos com a saúde e a adoção de medidas relacionadas à reorganização da atenção, decorreram mais de respostas a iniciativas nacionais do que de iniciativas próprias do nível local. Isto aponta a importância da definição de políticas por parte do nível central na condução de ações locais e, por outro lado, pode revelar as limitações da gestão municipal ou de sua insuficiência técnica na proposição de ações visando melhorias na atenção à saúde ${ }^{12}$. 
Ao analisar os gastos de recursos municipais, em estudos realizados no nordeste do país, verificou-se que estes se destinam prioritariamente à atenção básica ${ }^{13,25}$. No entanto, da forma como estes serviços são implantados, sem o suprimento necessário de recursos que garantam seu pleno funcionamento, levam à ineficiência na qualidade dos serviços. Um exemplo disso é o aumento do número de equipes de SF sem a manutenção de cobertura adequada à população, principalmente em grandes centros urbanos ${ }^{13,21}$.

Tendo como base as dificuldades enfrentadas quanto aos investimentos e gastos na área da saúde, verifica-se que o planejamento e a avaliação foram tidos como problemáticos em diferentes localidades do país ${ }^{8,10,12,14,26}$. O município não tem política de planejamento implantada, ou quando esta ocorre, se dá de forma incipiente ${ }^{10,25-27}$. Constatou-se que a alta rotatividade na ocupação dos cargos de gestão e a baixa qualificação formal para o exercício dessas atividades comprometem a operacionalização da descentralização no que diz respeito ao planejamento e gestão $0^{8,14}$.

Em vários municípios do Rio Grande do Norte verificou-se que o planejamento e a gestão dos recursos financeiros estão centralizados nas prefeituras. A concentração de ações, principalmente na questão orçamentária, está nas secretarias de finanças, o que decorre da ausência de estrutura nas de saúde. Esta situação faz com que o planejamento dos recursos, feito especificamente pelo contador, nem sempre atenda as necessidades da população, e que as secretarias de saúde, embora estejam amparadas pelos instrumentos de planejamento, tornem-se simples prestadoras de serviços ${ }^{25}$.

Outra fragilidade apontada em vários municípios é que os instrumentos de planejamento e gestão são limitados, ou muitas vezes, insuficientes, uma vez que, quando existem, apontam basicamente os objetivos e metas, focados nas exigências normativas sem retratarem a realidade local ${ }^{14,25,26}$. Houve também relato de descompasso entre o que havia sido planejado nos Planos Municipais de Saúde (PMS) e nas Programações Anuais de Saúde (PAS) e os resultados avaliados nos Relatórios Anuais de Gestão (RAG). Comparando-se estes instrumentos, as divergências eram grandes para o mesmo período, o que mostra a incompatibilidade entre o planejamento, a execução e a avaliação das ações ocorridas nos municípios estudados ${ }^{14}$.

Em relação à Programação Pactuada Integrada (PPI) como instrumento de compartilhamento da gestão, em dois municípios baianos verificou-se que a instância estadual tem um poder desigual na hora de pactuar metas, impondo condições que não condizem com a realidade local. O desenho e a forma como são decididas as questões na PPI contribuem para constranger a autonomia dos municípios, não favorecem a regionalização solidária, ignoram acordos e negociações e estimulam a competição entre os entes. A assimetria nas negociações e a falta de garantia do cumprimento dos acordos não favorecem uma postura mais solidária e cooperativa para a negociação dos municípios, os quais têm dificuldade de considerar interesses que extrapolem os seus limites territoriais ${ }^{26}$.

Além disso, nestes municípios, verificou-se desequilíbrio na pactuação de investimentos financeiros entre o fundo federal e o estadual, observada na insuficiência do estado em cumprir suas contrapartidas ${ }^{26}$. De acordo com a Lei $141 / 2012^{28}$ que regulamentou a Emenda Constitucional 29/2000 e dispõe sobre os valores mínimos a serem aplicados anualmente pela União, Estados e Municípios em ações e serviços de saúde, a União deve aplicar o montante correspondente ao valor empenhado no exercício financeiro anterior, acrescido de, no mínimo, o percentual correspondente à variação nominal do Produto Interno Bruto (PIB) ocorrida no ano anterior ao da lei orçamentária anual. Os Estados devem aplicar, no mínimo, 12\% e os Municípios 15\% do orçamento próprio. Um dos maiores entraves da descentralização tem sido o descumprimento do repasse financeiro por parte da União e dos Estados de acordo com percentuais denominados em lei, o que acaba fazendo com que os municípios tenham que aportar um percentual além do que está estabelecido.

Verificou-se em vários estudos que o processo decisório não é efetivamente compartilhado, visto que a esfera municipal detém menos recursos, e tem o poder reduzido, ficando subordinada às decisões das esferas federal e estadual. Há, portanto, distintos graus de poder entre os entes federados, que não os coloca em patamares semelhantes para que o compartilhamento aconteça ${ }^{8,19,26}$.

A efetividade dos instrumentos de gestão como mecanismo de planejamento, organização e pactuação da assistência nos municípios e regiões, para garantir o acesso da população aos diversos níveis de assistência, requereria um processo sistemático de acompanhamento da execução da programação, de aperfeiçoamento dos próprios instrumentos e processos de negociação. Entretanto, não existem instrumentos de avalia- 
ção, controle e acompanhamento sobre o desenvolvimento desse processo nas regiões. Desta forma, os municípios enfrentam dificuldades para executar as tarefas pactuadas, além de necessitar de avanços em relação à democratização dos processos de planejamento e programação local ${ }^{6,13,21}$.

Embora tenham sido apontados aspectos positivos quanto a iniciativas de interação entre a atenção básica e a média e alta complexidade $e^{6,12,18,21}$, também foi apontado que a ESF encontra dificuldades na referência para estes serviços ${ }^{6,13,21,23}$. Muitas vezes, o acesso aos serviços de maior densidade tecnológica, mesmo os pactuados, na prática, não ocorrem. Os municípios acabam vivenciando uma realidade na qual o SUS é universal, mas vive de cotas e limites, sendo difícil conseguir mantê-lo como um direito universal ${ }^{6,13}$. Isso se deve ao fato de que a pactuação de média e alta complexidade se constitui em um processo complicado, em que as regras e definições não estão claramente delimitadas nos instrumentos de planejamento e monitoramento. Em relação aos serviços de alta complexidade, tais instrumentos não existem ${ }^{21}$.

$\mathrm{Na}$ atenção de média complexidade, verificou-se demora para conseguir referência para especialidades e também dificuldades para agendamento de procedimentos especializados. Este entrave acarreta um grande número de encaminhamentos para Tratamento Fora do Domicílio (TFD). Quando o atendimento especializado é realizado, muitas vezes o retorno do especialista não é contrarreferenciado à ESF, o que evidencia esta fragilidade na relação entre a Atenção Básica $(\mathrm{AB})$ e a média complexidade ${ }^{6,13}$. Pesquisa realizada em um município da região metropolitana do Rio de Janeiro aponta que a sobrecarga de encaminhamentos para a atenção especializada pode estar atrelada à baixa resolutividade na APS ${ }^{7}$. Esta demanda elevada para a atenção de média complexidade evidencia dificuldades na gestão dos recursos disponíveis e estrangulamento da atenção especializada ${ }^{13}$.

A contratação de serviços da rede privada tem sido uma das estratégias para suprir deficiências de serviços de média e alta complexidade, o que os coloca em posição de domínio nos processos decisórios ${ }^{13}$. Os principais problemas vivenciados diretamente pelo hospital e pela atenção especializada incluem a baixa remuneração do SUS, considerada inadequada pelos prestadores, o aumento do preço dos insumos, além de limitações do número de atendimentos ${ }^{13,21}$.

A análise do processo de descentralização em áreas específicas, no município de São Paulo (SP), evidenciou falta de apoio político da própria gestão da Secretaria Municipal de Saúde (SMS-SP) para implementação de regiões de saúde, além da inexpressiva participação de atores estratégicos para sua viabilização ${ }^{8}$. Outros fatores que sinalizam dificuldades do processo de implementação de redes foram o caráter centralizador da SMS, a manutenção das estruturas político-administrativas independentes para a gestão da atenção básica e da assistência hospitalar, não assumindo a administração de ambulatórios e hospitais estaduais ${ }^{27}$.

Quanto à área de saúde bucal, verificou-se que a descentralização na (re)organização dos serviços odontológicos em municípios da Bahia apresentaram dificuldades quanto ao acolhimento para a humanização do acesso e a inexistência da garantia de término do tratamento ${ }^{29}$. Outro estudo apontou várias dificuldades para a construção da rede regionalizada de saúde bucal, como: falta de recursos materiais, em termos de quantidade e qualidade; restrição de espaço físico e de equipamentos; baixa arrecadação financeira em municípios de menor porte; fragilidade do serviço de apoio diagnóstico, com poucos equipamentos de RX; desarticulação no sistema de transporte intermunicipal de usuários; déficit de dados epidemiológicos e de registro nos sistemas de informação; e insuficiência no número de vagas oferecidas para média e alta complexidade ${ }^{23}$.

Quanto às ações de controle da hanseníase, em estudo realizado em nove municípios de Minas Gerais, observou-se que o serviço de referência teve dificuldade de entender e incorporar o seu novo papel de apoiar a $\mathrm{AB}$, e não mais de assumir integralmente o tratamento do paciente $^{30}$. Em relação ao tratamento da Tuberculose, em cinco municípios da região Sudeste e Nordeste foram encontradas dificuldades de acesso ao diagnóstico, e na organização da atenção, tanto na ESF quanto no ambulatório. Portanto, este fato demonstra que a alta cobertura da ESF não representa desempenho satisfatório para o acesso ao diagnóstico e tratamento desta doença ${ }^{31}$.

Ao avaliar a gestão descentralizada da assistência farmacêutica em dois municípios da Bahia, constatou-se que o processo de aquisição e distribuição de medicamentos não vem suprindo de forma suficiente as UBS, gerando insatisfação dos prescritores e gestores quanto à quantidade $\mathrm{e}$ tipos disponíveis nas UBS. Desta forma, o acesso do usuário aos medicamentos é uma problemática enfrentada pelos municípios, impedindo uma assistência à saúde integral e de qualidade ${ }^{32}$.

$\mathrm{Na}$ área de Vigilância Sanitária, o processo de descentralização encontra problemas crônicos 
nas três esferas de gestão, como a insuficiência de recursos humanos, em número e capacitação; deficiências de infraestrutura quanto a instalações, equipamentos, recursos materiais e financeiros; dificuldades na utilização do incentivo financeiro, interferências políticas, falta de planejamento das ações e de comunicação entre as três esferas de gestão $0^{33,34}$.

\section{Tecendo algumas considerações sobre perspectivas da descentralização}

As experiências de descentralização demonstraram que os municípios encontram-se em diferentes estágios e que esse processo tem sido determinado pela especificidade local e por avanços no processo de gestão de cada um.

Diversos desafios fazem parte do processo de consolidação do SUS, e requerem o aperfeiçoamento de competências técnicas e gerenciais. Esses desafios envolvem: definição das responsabilidades federativas, acesso e utilização de serviços especializados, construção de bases consistentes de planejamento regional, ampliação da equidade tanto no acesso quanto na qualidade dos serviços, e fortalecimento do controle social, entre outros ${ }^{20}$.

A descentralização, ao permitir a ampliação de transferências de recursos a estados e municípios, não garantiu o compartilhamento das funções e competências de gestão do sistema entre os entes federados. A autonomia dos municípios e a ausência de uma definição clara dos papéis a serem desempenhados pelo estado e união desencadeiam complexidade nas negociações em torno da descentralização, o que requer do nível federal e estadual capacidade de normatização, regulação do funcionamento e de monitoramento e avaliação do sistema ${ }^{35}$.

Além disso, é importante que os estados retomem seu papel de intermediário na relação município/governo federal e auxiliem os primeiros, principalmente em assumir a responsabilidade pela efetivação das regiões e redes de atenção à saúde, e também no desenvolvimento do Contrato Organizativo de Ação Pública (COAP), como instrumento de gestão potencial para viabilizar o desenvolvimento de uma rede interfederativa eficaz e sólida para o SUS. Para que o exercício da gestão interfederativa ocorra, torna-se imprescindível efetivar as Comissões Intergestoras Regionais (CIR) como espaços de discussão e tomada de decisão para, assim, construir uma política consensual e de corresponsabilidade pela regionalização da saúde.
A integração entre os diversos níveis de atendimento e as ações de integralidade e intersetorialidade são um grande desafio para a construção do SUS. O processo de municipalização radical resultou em um obstáculo quanto às diretrizes de hierarquização e regionalização da rede de serviços, sendo necessário desde o desenvolvimento de estratégias que articulem as reformas dos sistemas locais a iniciativas de cunho regional ${ }^{36}$.

A institucionalização de práticas mais articuladas, com clareza quanto às rotinas para o atendimento e os encaminhamentos de referência e contrarreferência dos usuários na rede, pode repercutir positivamente nos resultados da acessibilidade, para que o usuário não se perca pelo caminho e possa fazer um trajeto na rede de cuidados com respostas mais integrais e equânimes ${ }^{18}$.

A reorganização da atenção primária com vistas à universalidade do acesso a ações e serviços e à integralidade nas ações, implica também em que as demandas sejam respondidas em todas as instâncias de assistência. Para isso, é relevante a atuação dos profissionais da Atenção Básica no intuito de responder à maior parte dessas demandas e referenciar somente os casos específicos, além de melhorar a eficácia no sistema de monitoramento e regulação para otimização do acesso e do uso das tecnologias duras ${ }^{6}$. As tecnologias de saúde produzem o cuidado a partir de dimensões materiais e não materiais do fazer. Segundo Merhy ${ }^{37}$, as tecnologias duras são equipamentos e máquinas, as leve-duras são os saberes tecnológicos clínicos e epidemiológicos e as leves são os modos relacionais de agir dos profissionais na produção dos atos de saúde. Hoje, faz-se necessário dar sentido a todos os tipos de tecnologias para que as necessidades em saúde dos usuários sejam supridas.

Verifica-se a necessidade de estudos que analisem o processo de descentralização para os MPP, haja vista estes municípios terem importante representatividade no território brasileiro. No entanto, há poucos estudos específicos sobre a realidade destes municípios que possuem menor aporte financeiro, menos trabalhadores capacitados e pouco poder decisório nas políticas de saúde.

O financiamento foi apontado como uma barreira para o avanço da descentralização, pois esta não foi garantida por investimentos federais, tampouco estaduais, ficando o município com maior gasto e comprometimento do seu orçamento.

Poucos estudos analisaram o acesso aos serviços de média e alta complexidade, e os que o 
fizeram destacam as dificuldades de articulação dos municípios com estes serviços. Isto pode ter relação com a fragmentação e a desorganização de serviços de saúde devido à existência de sistemas isolados e sem intercomunicação, necessitando de maiores esforços para a efetivação do princípio da regionalização, com uma maior participação do estado com vistas ao enfrentamento desta lacuna assistencial.

Desta forma, faz-se necessário organizar o processo de regionalização para resolver as desigualdades presentes no acesso e na utilização dos serviços para que assim haja uma atenção à saúde mais integral, permitindo a integração de servi- ços, instituições e práticas no território que favoreçam a formação de arranjos mais cooperativos.

Faz-se necessário o diálogo, a reflexão e o agir articulado sobre a situação da saúde em nível local e regional. Essas discussões também devem alcançar espaços de participação popular de modo que promovam a sensibilização da comunidade e de seus representantes sobre a assistência à saúde no SUS.

Enfim, os elementos pontuados são necessários para que o SUS possa se constituir enquanto gestão compartilhada e rede interfederativa de saúde, consolidando-se como um sistema de saúde de qualidade, resolutivo e integral para o usuário.

\section{Colaboradores}

E Pinafo, BG Carvalho e EFPA Nunes trabalharam na concepção, análise, interpretação dos dados, redação do artigo e revisão.

\section{Agradecimentos}

A pesquisa contou com o apoio financeiro da Fundação Araucária: Apoio e Desenvolvimento Científico e Tecnológico do Paraná e Capes. 


\section{Referências}

1. Brasil. Ministério da Saúde (MS). Secretaria de Gestão Estratégica e Participativa. Decreto no 7.508, de 28 de junho de 2001. Regulamenta a Lei no 8.080 , de 19 de setembro de 1990, para dispor sobre a organização do Sistema Único de Saúde - SUS, o planejamento da saúde, a assistência à saúde e a articulação interfederativa, e dá outras providências. Diário Oficial da União 2011; 29 jun.

2. Lima LD, Viana ALD'A, Machado CV. A regionalização da saúde no Brasil: condicionantes e desafios. In: Scatena, JHG, Kehrig RT, Spinelli MA, organizadores. Regiões de Saúde: diversidade e processo de regionalização em Mato Grosso. São Paulo: Hucitec; 2014. p. 565

3. Viana ALd'A, Lima LD. O processo de regionalização na saúde: contextos, condicionantes e papel das Comissões Intergestores Bipartites. In: Viana ALd'A, Lima LD, organizadores. Regionalização e relações federativas na política de saúde do Brasil. Rio de Janeiro: Contracapa; 2011.p. 11-24.

4. Souza C. Governos e sociedades locais em contextos de desigualdades e de descentralização. Cien Saude Colet 2002; 7(3):431-442.

5. Blumm M, Souza C. Autonomia política local: uma revisão de literatura. Trabalho apresentado no XXIII Encontro Nacional de Pós Graduação em Administração (ENAMPAD), 1999; Anais 1 CD-ROM.

6. Viegas SMF, Penna CMM. O SUS é universal, mas vivemos de cotas. Cien Saude Colet 2013; 18(1):181-190.

7. Monnerat GL, Senna MCM, Souza RG. A reorganização dos serviços de saúde no cenário local. Cien Saude Colet 2002; 7(3):509-521.

8. Spedo SM, Pinto NRS, Tanaka OY. A regionalização intermunicipal do Sistema Único de Saúde (SUS): um estudo de caso do município de São Paulo-SP, Brasil. Saúde Soc 2010; 19(3):533-546.

9. Arksey H, O’Malley L. Scoping studies: towards a methodological framework. Int J Soc Res Methodol 2005; 8(1):19-32.

10. Souza MKB, Melo CMM. Perspectiva de enfermeiras gestoras acerca da gestão municipal da saúde. Rev Enfermagem UERJ 2008; 16(1):20-25.

11. Borges DF, Fernandes KC. A descentralização das ações e serviços de saúde do SUS na cidade de Natal: democratização ou privatização. RAP 2002; 36(4):565-585.

12. Vieira-da-Silva LMV, Hartz ZMA, Chaves SCL, Silva GAP, Paim JS. Análise da implantação da gestão descentralizada em saúde: estudo comparado de cinco casos na Bahia, Brasil. Cad Saude Publica 2007; 23(2):355-370.

13. Coriolano MWL, Albuquerque GA, Araújo NS, Oliveira MA, Lima MM. Vivenciando o processo de municipalização do SUS no município de Juazeiro do Norte (CE). Cien Saude Colet 2010; 15(5):2447-2454.

14. Vieira JMR, Garnelo L, Hortale VA. Análise da atenção básica em cinco municípios da Amazônia Ocidental, com ênfase no Programa Saúde da Família. Saúde Soc 2010; 19(4):852-865.

15. Vieira V, Andrade FR, Castro CGJ, Bighetti TI, Narvai PC. Municipalização de serviços de saúde segundo profissionais de saúde bucal em um município do interior do estado de São Paulo, Brasil. Saúde Soc 2013; 22(3):795-803.
16. Costa NR, Pinto LF. Avaliação de programa de atenção à saúde: incentivo à oferta de atenção ambulatorial e a experiência da descentralização no Brasil. Cien Saude Colet 2002; 7(4):907-923.

17. Arretche M, Marques E. Municipalização da saúde no Brasil: diferenças regionais, poder do voto e estratégias de governo. Cien Saude Colet 2002; 7(3):455-479.

18. Cunha ABO, Vieira-da-Silva LM. Acessibilidade aos serviços de saúde em um município do Estado da Bahia, Brasil, em gestão plena do sistema. Cad Saude Publica 2010; 26(4):725-737.

19. Pinto NRD, Tanaka OY, Spedo SM. Política de saúde e gestão no processo de (re)construção do SUS em município de grande porte: um estudo de caso de São Paulo, Brasil. Cad Saude Publica 2009; 25(4):927-938.

20. Fleury S, Ouverney ALM, Kronemberger TS, Zani FB. Governança local no sistema descentralizado de saúde no Brasil. Rev Panam Salud Publica 2010; 28(6):446455.

21. Almeida PF, Giovanella L, Mendonça MHM, Escorel S. Desafios à coordenação dos cuidados em saúde: estratégias de integração entre níveis assistenciais em grandes centros urbanos. Cad Saude Publica 2010; 26(2):286-298.

22. Barros SG, Vianna MIP, Chaves SCL. Descentralização da saúde e utilização de serviços odontológicos em 11 municípios da Bahia. Rev. Baiana de Saúde Pública 2009; 33(3):372-387.

23. Mello ALSF, Andrade SR, Moysés SJ, Erdmann AL. Saúde bucal na rede de atenção e processo de regionalização. Cien Saude Colet 2014; 19(1):205-214.

24. Balista SRR, Santiago SM, Corrêa Filho HR. A descentralização da vigilância da saúde do trabalhador no Município de Campinas, São Paulo, Brasil: uma avaliação do processo. Cad Saude Publica 2011; 27(4):759768.

25. Leite VR, Lima KC, Vasconcelos CM. Financiamento, gasto público e gestão dos recursos em saúde: o cenário de um estado brasileiro. Cien Saude Colet 2012; 17(7):1849-1856.

26. Molesini, JA, Formigli VLA, Guimarães MCL, Melo CMM. Programação pactuada integrada e gestão compartilhada do SUS. Rev. Baiana de Saúde Pública 2010; 34(3):623-638.

27. Spedo SM, Tanaka OY, Pinto NRS. O desafio da descentralização do Sistema Único de Saúde em município de grande porte: o caso de São Paulo, Brasil. Cad Saude Publica 2009; 25(8):1781-1790.

28. Brasil. Lei Complementar $n^{\circ} 141$, de 13 de Janeiro de 2012. Regulamenta o $\$ 3$ o do art. 198 da Constituição Federal para dispor sobre os valores mínimos a serem aplicados anualmente pela União, Estados, Distrito Federal e Municípios em ações e serviços públicos de saúde; estabelece os critérios de rateio dos recursos de transferências para a saúde e as normas de fiscalização, avaliação e controle das despesas com saúde nas 3 (três) esferas de governo; revoga dispositivos das Leis nos 8.080, de 19 de setembro de 1990, e 8.689, de 27 de julho de 1993; e dá outras providências. Brasília. Diário Oficial da União 2012; 13 jan. 
29. Chaves SCL, Vieira-da-Silva LM. Atenção à saúde bucal e a descentralização da saúde no Brasil: estudo de dois casos exemplares no Estado da Bahia. Cad Saude Publica 2007; 23(5):1119-1131.

30. Lanza FM, Lana FCF. Descentralização das ações de controle da hanseníase na microrregião de Almenara, Minas Gerais. Rev. Latino-Am. Enfermagem 2011; 19(1):187-194.

31. Scatena LM, Villa TCS, Ruffino Netto A, Kritski AL, Figueiredo TMRM, Vendramini SHF, Assis MMA, Motta MCS. Dificuldades de acesso a serviços de saúde para diagnóstico de tuberculose em municípios do Brasil. Rev Saude Publica 2009; 43(3):389-397.

32. Barreto JL, Guimarães MCL. Avaliação da gestão descentralizada da assistência farmacêutica básica em municípios baianos, Brasil. Cad Saude Publica 2010; 26(6):1207-1220.

33. Juliano IA, Assis MMA. A vigilância sanitária em Feira de Santana no rpocesso de descentralização da saúde (1998-2000). Cien Saude Colet 2004; 9(2):493-505.

34. Marangon MS, Scatena JHG, Costa EA. Vigilância sanitária: estratégias para sua descentralização em Mato Grosso, 1996-2005. Cien Saude Colet 2010; 15(Supl. 3):3587-3601.

35. Levcovitz E, Yamamoto EK, Silva LAD. Notas sobre a crise de financiamento do setor público no âmbito do SUDS. Saúde Debate 1989; (27):12-17.

36. Pasche DF, Righi LB,Thome HI,Stolz ED. Paradoxos das políticas de descentralização de saúde no Brasil. Rev Panam Salud Publica 2006; 20(6):416-422.

37. Merhy EE. Um dos grandes desafios para os gestores do SUS: apostar em novos modos de fabricar os modelos de atenção. In: Merhy EE, Magalhães Júnior HM, Rimoli J, Franco TB, Bueno WS, organizadores. O trabalho em saúde: olhando e experienciando o SUS no cotidiano. $3^{\text {a }}$ ed. São Paulo: Hucitec; 2006. p. 15-36.

Artigo apresentado em 16/06/2015

Aprovado em 09/11/2015

Versão final apresentada em 11/11/2015 